\title{
Effects of Threatening Communications and Mothers' Health Beliefs on Weight Change in Obese Children
}

\author{
John P. Kirscht, ${ }^{1}$ Marshall H. Becker, ${ }^{1}$ \\ Don P. Haefner, ${ }^{1}$ and Lois A. Maiman ${ }^{2}$ \\ Accepted for publication: January 6, 1978
}

A field experiment was designed to test the effects on weight change in obese children of (1) communications varying in threat and (2) health beliefs of the child's mother, in terms of perceived vulnerability to health threats, the efficacy of taking actions, and barriers to acting. Treatments involved messages containing more or less threatening material regarding obesity, plus a control (no communication) condition. Beliefs were assessed by means of personal interviews. All mothers and children received dietary counseling and were scheduled for four follow-up visits, which included weighing. The 182 participants were from low-income areas served by a large hospital pediatric clinic. High-threat messages yielded the most consistent weight loss, followed by low threat and control. In addition, both general beliefs concerning health and specific beliefs about obesity and dieting predicted weight loss. Both messages and mothers' beliefs acted together on keeping appointments and making efforts to comply. Results are discussed in terms of the effects of threats and health belief-behavior linkages.

KEY WORDS: childhood obesity; health belief model; obesity; threatening communications.

\section{INTRODUCTION}

Despite recent controversy about belief-behavior relationships (Wicker, 1969; Gross and Niman, 1975), there is evidence that persuasion approaches to change are effective in bringing about behavioral changes, mediated through

This investigation was supported by Grants HL 5P17HL14207 and HL 18045 from the National Heart and Lung Institute.

${ }^{1}$ Department of Health Behavior. University of Michigan, Ann Arbor, Michigan 48109.

${ }^{2}$ Department of Social and Administrative Pharmacy, University of Minnesota, Minneapolis, Minnesota 55455. 
cognitive processes (Kelman, 1974). The critical questions are: under what circumstances are beliefs associated with behavior? what sorts of beliefs are involved? and what approaches to belief change are more likely to result in behavioral outcomes?

Several of the conceptual approaches arguing for belief-behavior consistency make belief specificity a key issue (Rokeach, 1969; Fishbein and Ajzen, 1975), noting, with some validity, that the level of generality at which beliefs or attitudes are assessed has frequently been quite disparate from the specificity of the measured behavioral outcomes. The issue of specificity raises the problem of what aspects of belief structures are called forth with respect to a particular behavior and what sets of beliefs provide an interpretive reference for a particular situation in which behavior occurs.

Attempts to formulate a cognitive approach to health-related behavior are summarized by Rosenstock (1974). This framework for explaining personal decisions to act with respect to prevention, illness, and the sick role comprises beliefs about health threats (in terms of perceived probability of an illness or condition occurring and the perceived negative consequences of that condition) and about the value of actions with respect to warding off or alleviating the threat, tempered by the psychological costs of acting. Rogers (1975) described this approach as "protection theory." It holds that specific beliefs are the most immediate antecedents of decisions to act, although such beliefs are thought to be related to more general perceptions of health, as well as to sociocultural factors.

Several investigators have been interested in the effects on health-related behavior of attempts to modify beliefs about the nature of health threats, and the value of acting (e.g., Haefner and Kirscht, 1970; see also Leventhal, 1970, 1973). It is recognized that this is an indirect approach to behavior change (Crawford, 1974).

A significant portion of research on persuasion relative to health has involved the issue of threat or fear arousal. Most health topics are, at least implicitly, threatening to the individual, hence the affinity of research on fear for health issues. Although the study of threatening communications has yielded a conflicting array of findings (Leventhal, 1965, 1970; Janis, 1967; Higbee, 1969), the weight of evidence suggests that a high level of threat is often effective in changing beliefs and behavior (McGuire, 1969). Such effects appear to be conditioned by the sense of personal vulnerability to the threat and the availability of efficacious actions (Leventhal, 1970). Threats may affect the individual's beliefs about the presence of danger, especially where risk is not recognized or not taken seriously enough. Communications with differing levels of threat may be differentially effective in bringing belief dimensions into salience.

Recent evidence for the utility of the health belief framework in relation to both behavioral intention and fear-arousing communication is presented by 
Rogers and Mewborn (1976), who found that more threatening messages modified perceptions of the severity of health problems. Perceived efficacy of acting was the best predictor of behavioral intention and appeared to mediate attitude change toward the behavior in the presence of threatening communications.

The present study sought to assess the effects of (1) communications with varying degrees of threat and (2) health belief variables on weight change in obese children (where the beliefs of mothers of the children were the focus of interest). Specifically, the study was designed to test the hypothesis that a higher level of threat in a communication about the health effects of obesity would achieve more behavioral compliance with dietary recommendations than would a lower level of threat, which, in turn, was expected to affect compliance more than no communication. In addition, the study sought to assess the predictability of the child's weight change from the mother's initial beliefs concerning health threats to the child, obesity as a health problem, and the effectiveness of dieting. Past research had indicated that mothers' health beliefs did predict compliance with medical recommendations for an acute illness in children (Becker et al., 1972) and the investigation attempted to extend these findings to a chronic health condition whose treatment involves alteration of daily living habits. Obesity in children is widely recognized as a major health problem (Mayer, 1975), particularly because of its relationship to severe adult obesity and association with mortality and morbidity from diabetes and cardiovascular disease (American Academy of Pediatrics, 1967).

\section{METHOD: DESIGN AND SUBJECTS}

A three-group design was used, with random assignment to groups. The two experimental treatments involved oral and written messages: (1) a more threatening message and booklet concerning obesity and its potential negative health consequences and (2) a less threatening message and booklet. The former emphasized the seriousness of ill-health conditions related to obesity (e.g., "The overweight child is likely to become an obese adult... Heart attacks, strokes, high blood pressure, and diabetes happen a lot more often to obese people - add it all up and you get a shorter life expectancy."). In the latter messages, less graphic words were used and more general statements substituted (e.g., "There are health problems related to obesity, most of which take years to develop. Being obese can interfere with things that people want to do .... All in all, obese people are more likely to have health problems."). The two versions were of the same length and structure and, in both cases, contained reassuring information about the benefits of weight loss. The third (control) group received no messages.

The study was conducted in the large ambulatory pediatric clinic at a major teaching hospital. From July 1973 to July 1975 mothers of children 
newly identified as obese $(N=199)$ were referred to the clinic dietitian for instruction and a weight reduction plan, and then invited to participate in the study. Only persons responsible for the child's daily care, and for bringing the child to the clinic when necessary, were included.

An initial interview, separate from the clinic meeting with the dietitian, was conducted with each participant, and included questions about health beliefs: concern about health, the threat of illnesses, efficacy of actions, and views about obesity and dieting. This was followed by random assignment to groups, with messages given to those in the two experimental groups. Mothers and children were subsequently scheduled for meetings with the dietitian about every 2 weeks. A weight plan was tailored to the individual child, but always involved calorie reduction in total intake, with instructions about food purchases and preparation.

Respondents (including two grandmothers) ranged in age from 17 to 62 years (mean 37.2) and children from 19 months to 17 years (mean 11.5). More than twice as many of the children were female, and all but 11 were black. The clinic serves a predominantly poor population, and this was reflected in the low levels of education and income in the sample. On entry into the study, the mean weight of the children was $147.4 \mathrm{lb}$, representing an average of $54 \%$ over the ideal weight determined by the dietitian.

Of the 199 potential subjects, 16 were lost due to scheduling conflicts and errors in maintaining records; one mother refused to participate. Eighteen of the 182 remaining subjects did not return for the first follow-up counseling and measurement visit, and were therefore dropped from the study. At this follow-up visit (FUV), the high-threat, low-threat, and control groups contained 56,55 , and 53 subjects, respectively $(N=164)$. An additional 19 subjects failed to return for the second FUV $(N=145), 20$ more by the third FUV $(N=125)$, and an added 12 by the final visit $(N=113)$.

Analyses of the dropouts revealed that attrition rates, weight change, and belief scores did not differ significantly across experimental groups and therefore could not account for any group differences observed, and dropout mean belief scores were somewhat (but not significantly) lower than those of the continuing subjects (suggesting the relevance of these attitudes to continued participation). It was therefore concluded that sample mortality probably had little influence on the findings.

The three groups did not differ significantly in terms of amount overweight on initial visit, each group averaging $54 \%$ over ideal weight. Upon assessment, the children were, of course, overweight to varying extents; initial weights varied from 50 to $346 \mathrm{lb}$ and ranged from $9 \%$ to $188 \%$ over ideal weight. To achieve standardization across individuals, the major dependent variable was the ratio of cumulative weight change between visits to weight on initial visits. Correlations of this variable with several other measures of weight change (e.g., absolute weight change, ratio of weight change to predicted weight loss, and 
ratio of weight change to estimated ideal weight) were found never to fall below 0.970 .

\section{RESULTS}

\section{Reactions to the Messages}

An attempt was made to assess participants' reactions to the communications. Immediately after the presentations, a short adjective list was administered, and subjects were asked to indicate how they felt, using three ordered response categories. Very few differences appeared between the two threat groups on fear-related words, although the high-threat subjects scored significantly higher on the word "afraid" than the low-threat group $(t=1.99,109 \mathrm{df})$. The adjectives did not cluster together as expected from prior studies, primarily with college students, suggesting that this approach was not appropriate for the group studied. However, indirect evidence was available from a short questionnaire administered at the first FUV. This interview dealt principally with reactions to the diet but included an item on concern about the child's health. There was a significant difference among groups in response to this item, with the highthreat groups showing greatest concern, followed by the low-threat and control groups (mean scores in order were $4.40,4.08$, and $3.57, F=3.76,2$ and 152 df). It will be noted later that concern with the child's health was a signficant predictor of subsequent weight loss.

\section{Experimental Group and Weight Change}

Mean cumulative weight change scores are entered in Table I. It can be seen that, at each follow-up visit, the high-threat group had lost more weight,

Table I. Mean Cumulative Weight Change by Group $a$

\begin{tabular}{lcccc}
\hline & \multicolumn{5}{c}{ Follow-up visit } \\
\cline { 2 - 5 } \multicolumn{1}{c}{ Group } & 1 & 2 & 3 & 4 \\
\hline High threat & -1.827 & -2.158 & -2.556 & -2.591 \\
Low threat & -0.817 & -1.259 & -1.024 & -1.456 \\
Control & +0.091 & -0.283 & -0.163 & -0.118 \\
Total $N$ & $(164)$ & $(145)$ & $(125)$ & $(113)$ \\
$F$ for ANOVA & 20.83 & 11.23 & 9.97 & 7.16 \\
& $p<0.001$ & $p<0.001$ & $p<0.001$ & $p<0.002$ \\
\hline aWeight change & $=$ current & weight - initial weight divided by \\
initial weight $x$ & 100, with weight change prorated to standard \\
14-day periods.
\end{tabular}


Table II. Comparisons Among Mean Weight Change Scores (Table I) by Group at FUV 1 and FUV 4 ( $t$ Tests)

\begin{tabular}{lcc}
\hline Groups compared & FUV 1 & FUV 4 \\
\hline High threat vs. & $t=3.216^{a}$ & $t=1.651$ \\
low threat & $109 \mathrm{df}$ & $74 \mathrm{df}$ \\
Low threat vs. & $t=3.272^{a}$ & $t=2.157^{b}$ \\
control & $106 \mathrm{df}$ & $72 \mathrm{df}$ \\
High threat vs. & $t=6.510^{a}$ & $t=3.755^{a}$ \\
control & $107 \mathrm{df}$ & $74 \mathrm{df}$ \\
\hline$a_{p}<0.01$. & & \\
$b_{p}<0.05$. & &
\end{tabular}

followed by the low-threat group and controls. These ratio scores represent actual changes of $-2.9 \mathrm{lb},-1.2 \mathrm{lb}$, and $0.1 \mathrm{lb}$ over the first 2-week period. For the entìre period of the study, which averaged 61 days per child, the mean weight change was $-4.1 \mathrm{lb},-2.1 \mathrm{lb}$, and $-0.2 \mathrm{lb}$ for the three groups. At each FUV, differences among the groups were significant. Controls actually gained weight, on the average, in three of the four periods. Those in the high-threat group showed the most consistent weight loss. Table II contains group-by-group comparisons, using $t$ tests, at FUV 1 and FUV 4; the only difference not reaching significance was that between the high-and low-threat groups at time 4 .

\section{Initial Health Beliefs and Weight Change}

Items pertaining to particular health belief dimensions were combined into indexes on the basis of content and interitem relationships. The major indexes dealt with (1) concern about the child's health (e.g., "how much do you worry about _ 's health"); (2) perceived threat to the child of each illness on a list both in terms of susceptibility ("how much chance is there - could ever get asthma") and severity ("how worried would you be if —- got rheumatic fever"); (3) the health threat of obesity ("how serious a problem is ___'s overweight"); (4) benefits of medical treatment for illness ("how much can a doctor help when a child has mumps"); (5) the benefits of the diet in relation to obesity ("how much will the diet help the problem");6) anticipation of following the diet ("how much trouble will it be to keep — on the diet"). Internal consistency for these indexes ranged from 0.47 to 0.96 , with the measures of health concern and threat yielding the highest consistencies and the benefits and anticipation indexes showing lowest consistencies. Several other potential indexes were discarded because of low reliability.

Correlations of the belief measure with weight change at FUV 1 and FUV 4 are presented in Table III. Most of the belief measures yielded significant positive correlations with weight change, except for the indexes of medical 
Table III. Correlations (Product Moment) of Belief Indexes and Social Characteristics with Weight Change at First and Last Follow-up Visits

\begin{tabular}{|c|c|c|c|c|}
\hline \multirow[b]{3}{*}{ Index } & \multicolumn{4}{|c|}{ Follow-up visits } \\
\hline & \multicolumn{2}{|l|}{1} & \multicolumn{2}{|l|}{4} \\
\hline & $r$ & $\beta^{a}$ & $r$ & $\beta^{a}$ \\
\hline $\begin{array}{l}\text { Concern with child's health } \\
\text { Illiess threat }\end{array}$ & $\left.\begin{array}{l}0.48^{b} \\
0.54^{b}\end{array}\right\}$ & $0.43^{b}$ & $\left.\begin{array}{l}0.27 b \\
0.35 b\end{array}\right\}$ & 0.02 \\
\hline Medical benefits for child & -0.02 & -0.10 & -0.02 & -0.15 \\
\hline Obesity as a health threat & $0.54 b$ & 0.17 & $0.40^{b}$ & $0.35^{b}$ \\
\hline $\begin{array}{l}\text { Benefits of dieting } \\
\text { Difficulties of dieting }\end{array}$ & $\left.\begin{array}{r}-0.04 \\
0.27 b\end{array}\right\}$ & $0.14^{b}$ & $\left.\begin{array}{l}0.10 \\
0.24 b\end{array}\right\}$ & $0.25^{b}$ \\
\hline Family income & 0.08 & & 0.01 & \\
\hline Mother's education & 0.06 & & -0.06 & \\
\hline Number of children in home & 0.02 & & 0.00 & \\
\hline Age of child & 0.13 & & $0.22^{b}$ & \\
\hline
\end{tabular}

$a_{\text {The }} \beta$ values from stepwise multiple regression. The first two indexes were combined into a measure of overall health concern and the last two into an index of overall efficacy of dieting. For the first FUV, $R=0.641$; for the last FUV, $R=0.51$.

$b_{p}<0.05$.

benefits and dietary benefits. Although the relationships were attenuated by the fourth visit, many of the correlations were still significant. In addition, multiple regressions were calculated, using the belief variables as predictors and weight change as the dependent variable. In general, the $\beta$ coefficients were comparable with the correlations; general beliefs concerning the child's health problems were the best predictors, especially at the first FUV, while weight-specific beliefs were relatively more important at the later FUV. Over $40 \%$ of the variance in weight change scores at FUV 1 could be accounted for by the belief measure $(R=0.641)$.

\section{Personal Characteristics}

Family income, mother's education, and number of children in the household were not correlated with weight change at any of the visits. The age of the child, however, yielded positive relationships to weight change (Table III); the direction indicated that older children were more successful in losing weight than were younger children, possibly reflecting peer influence toward the older child's cooperation with the diet. When age of the child was controlled, there was no change in the health belief-weight change relationships. These results suggest that the belief-behavior associations were not an artifact of social group differences. Degree of initial overweight was also examined as a possible contributor to spurious belief-behavior relationships; however, this measure was unrelated to weight change scores. 


\section{Experimental Treatments and Beliefs in Relation to Weight Change}

The messages about obesity contributed to weight loss independently of initial health beliefs, as indicated by two kinds of analyses: (1) A multiple regression of weight change on the belief indexes with the experimental treatment introduced as a dummy variable; treatment was significantly related to weight change but the belief measures also continued to be significant predictors. (2) Analyses of variance on the weight change scores with both experimental treatment and dichotomized belief indexes included as classification variables; both produced significant main effects while no interactions reached significance (Table IV is an example).

Further comparisons indicate that, among those whose beliefs about illness were low, participants in the high-threat group lost significantly more weight than controls $(t=4.431,52 \mathrm{df}, p<0.01)$. At the same time, control subjects with an initially high level of beliefs also lost significantly more than controls with low beliefs $(t=2.8156,49 \mathrm{df}, p<0.01)$.

\section{Some Mediating Effects}

The dietary counseling visits themselves apparently had an effect on weight. Keeping reappointments (within a week of schedule) correlated positively with weight loss at each follow-up visit. In the high-threat group $85 \%$ kept the first scheduled visit, while $65 \%$ of the low-threat group and only $60 \%$ of the controls appeared on schedule (for these differences, $\chi^{2}=9.25,2 \mathrm{df}, p<0.01$ ). Further, although the overall rate of dropping out varied little among the groups, the

Table IV. Mean Cumulative Weight Change to First Follow-up Visit, by Group and Initial Health Threat Score $^{a}$

\begin{tabular}{lcc}
\hline & \multicolumn{2}{c}{$\begin{array}{c}\text { Health threat } \\
\text { score }\end{array}$} \\
\cline { 2 - 3 } Group & Low & High \\
\hline High threat & -1.642 & -1.930 \\
Low threat & -0.491 & -1.270 \\
Control & +0.440 & -0.609 \\
$N$ 's & $(86)$ & $(75)$ \\
\hline$a$ Subjects were divided as closely \\
as possible at the median score \\
on the index of illness threat. \\
Overall ANOVA yielded an $F=$ \\
14.71 for groups (2, 160 df), \\
$p<0.01,6.516$ for beliefs $(1$, \\
$160 \mathrm{df}), p<0.02$, and $1.499(2$, \\
$160 \mathrm{df}), \mathrm{NS}$, for interaction.
\end{tabular}


controls who dropped after the first FUV were more likely to have gained weight than those who dropped out of the high- and low-threat groups $(t=2.29,17$ df, $p<0.05$ ).

\section{DISCUSSION}

Weight loss by the child was strongly related to the experimental conditions, with the high-threat message yielding the most consistent and positive results. Even though the effects tapered off later in the study, the interventions continued to have an impact throughout the period of follow-up (the median time period for the participants in all four FUVs was 61 days). The high-threat communication was more effective than low threat, quite possibly because it called attention to a health problem that may not have been seen as important or serious initially by the group studied. Although the dietitian had discussed the weight situation with everyone in the study, the communications appear to have added emphasis and reinforcement.

Some of the beliefs predictive of weight loss were nonspecific and seem to reflect willingness to act with respect to health matters. These general measures were also significantly associated with appointment keeping in the pediatric clinic over a year's period, excluding the obesity clinic visits. There is differentiation of beliefs, however: (1) beliefs specific to the obesity problem yielded higher predictions of weight loss at later FUVs, suggesting that specific reasons for dieting may sustain the behaviors somewhat better; and (2) perceived value of medical care was a significant predictor of general clinic appointment keeping but was unrelated to weight loss. Care for obesity is, of course, different from the usual medical care for illnesses.

It is reasonable to believe that the communications served a cue function in making the beliefs salient and in increasing the willingness to act. While belief change data were not available, it is quite possible that the high-threat message bolstered the health-related beliefs of those initially low. Even so, it is doubtful that beliefs alone or messages alone produced weight loss. They did make it more likely the mother would keep scheduled appointments and make initial efforts to comply. This in turn provided opportunities for reinforcement by the dietitian, the mother, and the child.

In this study, weight changes were modest and the issue of clinical significance was not addressed. However, in the initial time period, those in the highthreat group averaged a loss of nearly $3 \mathrm{lb}$. For the entire term of the study, this group lost about $0.5 \mathrm{lb}$ per week; $20 \%$ achieved losses of $1 \mathrm{lb}$ per week or more. Only two participants in the control group lost at that rate. Differential weight change is the major point of the findings.

To what extent are the findings from this study generalizable? The population represented was rather homogeneous, being largely female, less educated, and black. However, we believe that the connections found between beliefs and 
behavior do have external validity, particularly since their role has been demonstrated across a variety of situations (Becker and Maiman, 1975). The communication effects do need replication and extension. Dabbs and Leventhal (1966) noted that persons with low self-esteem were less likely to respond adaptively in the face of threat. Self-esteem is known to be related positively to socioeconomic status. However, some research indicates that people of lower SES are more likely to follow recommendations presented in a threatening context (Haefner, 1965). Furthermore, many of the participants in this study clearly believed in the efficacy of their own actions, in spite of what might seem to be objectively poor circumstances. Taken together, the findings point to the effectiveness of threatening communications that deal with areas in which there may be too low a sense of seriousness but where persons are already somewhat disposed to act (in this instance, by showing up at the obesity clinic) and where specific paths of action are available (Leventhal, 1970).

\section{REFERENCES}

American Academy of Pediatrics (1967). Obesity in childhood. Pediatrics 40: 455-467.

Becker, M., and Maiman, L. (1975). Sociobehavioral determinants of compliance with health and medical care recommendations. Med. Care 13: 10-24.

Becker, M., Drachman, R., and Kirscht, J. (1972). Predicting mothers' compliance with pediatric medical regimens. J. Pediat. 81: 843-854.

Crawford, T. (1974). Theories of attitude change and the "beyond family planning debate." The case for the persuasion approach in population policy. J. Soc. Issues 30: 211-233.

Dabbs, J., and Leventhal, H. (1966). Effects of varying the recommendations in a feararousing communication. J. Personal. Soc. Psychol. 4: 525-531.

Fishbein, M., and Ajzen, I. (1975). Belief, Attitude, Intention, and Behavior, AddisonWesley, Reading, Mass., 1975.

Gross, S., and Niman, C. (1975). Attitude-behavior consistency: A review. Publ. Opinion Quart. 39: 358-368.

Haefner, D. (1965). Arousing fear in dental health education. J: Publ. Health Dent. 25 : $140-146$.

Haefner, D., and Kirscht, J. (1970). Motivational and behavioral effects of modifying health beliefs. Publ. Health Rep. 85: 478-484.

Higbee, K. (1969). Fifteen years of fear arousal: Research on threat appeals, 1953-1968. Psychol. Bull. 72: 426-444.

Janis, I. (1967). Effects of fear arousal on attitude change: Recent developments in theory and experimental research. In Berkowitz, L. (ed.), Advances in Experimental Social Psychology, Vol. 3, Academic Press, New York.

Kelman, H. (1974). Attitudes are alive and well and gainfully employed in the sphere of action. Am. Psychol. 29: 310-324.

Leventhal, H. (1965). Feat communications in the acceptance of preventive health practices. Bull. N.Y. Acad. Med. 41: 1144-1168.

Leventhal, H. (1970). Findings and theory in the study of fear communications. In Berkowitz, L. (ed.), Advances in Experimental Social Psychology, Vol. 5, Academic Press, New York, 1970.

Leventhal, H. (1973). Changing attitudes and habits to reduce risk factors in chronic disease. Am. J. Cardiol. 31: 571-581.

Mayer, J. (1975). Obesity during childhood. In Winick, M. (ed.), Childhood Obesity, Wiley, New York, 1975. 
McGuire, W. (1969). The nature of attitudes and attitude change. In Lindzey, G., and Aronson, E. (eds.), Handbook of Social Psychology, Vol. 3, 2nd ed., AddisonWesley, Reading, Mass., 1969.

Rogers, R. (1975). A protection motivation theory of fear appeals and attitude change. J. Psychol. 91: 93-114.

Rogers, R., and Mewborn, C. (1976). Fear appeals and attitude change. Effects of a threat's noxiousness, probability of occurrence, and the efficacy of coping responses. $J$. Personal. Soc. Psychol. 34: 54-61.

Rokeach, M. (1969). Beliefs, Attitudes, and Values, Jossey-Bass, San Francisco, 1969. Rosenstock, I. (1974). Historical origins of the health belief model. Health Educ. Monogr. 2: $328-335$.

Wicker, A. (1969). Attitudes vs. actions: The relationship of verbal and overt behavioral responses to attitude objects. J. Soc. Issues 24: 41-78. 